\title{
Pre and Post emergence Cotton Herbicides in Varying Rainfall Patterns
}

\author{
Ambati Ravinder Raju ${ }^{1}$, Shilpa Rananware ${ }^{1}$ and Rachana Deshmukh ${ }^{2}$ \\ ${ }^{1}$ Dr. P. D. K. V., Akola, India \\ ${ }^{2}$ ICAR-Central Institute for Cotton Research, Nagpur-440010, MS, India \\ *Corresponding author
}

\section{Keyw ords}

Fenoxoprop-methyl, Glyphosate,

Propaquizafop, Pyrithiobac sodium, Quizalopfop -ethyl

\section{Article Info}

\section{Accepted:}

20 July 2018

Available Online:

10 August 2018

\section{A B S T R A C T}

A field experiment was conducted in vertisols in varying rainfall patterns during 2013, 2014 seasons at ICAR-Central Institute for Cotton Research farm, Nagpur. The main objective of this study was to advice the farmers in varying rainfall patterns on reduce their weed management cost through early post emergence graminicides. Farmers should carefully follow weather CICR weather advisory on cotton cultivation a weekly web bulletin. In a year of weak monsoon farmers should go for direct sowing of cotton whenever $60 \mathrm{~mm}$ rainfall is received without any pre emergence residual herbicide application as it does not work in desiccating weather conditions under seedling droughts. The year may begin with very good pre monsoon rains farmers have only one option glyphosate @1.0 kg a.i. ha ${ }^{-1}$ as PPF application on emerged weeds before or within three days after planting of cotton or before cotton seedling emergence. The year might have began with very good monsoon followed by weakened monsoon with late seedling drought where normal interculture operations can take care of weeds and conserve soil moisture followed by limited hand weeding with available family/hired labour. However, revival of monsoon with continuous heavy rains farmers can use early post emergence application of propaquizafop on 3-4 weeks old grassy weeds or pyrithiobac sodium against broad leaf weeds or a tank mixture on clear sunny day for atleast 3-4 hrs. In case of late active continuous monsoon rains after squaring stage of cotton young weeds are difficult to be removed by intercultural operations or hand weeding operations if allowed to grown consume all the top dressed urea. Therefore, farmers may use glyphosate $2 \mathrm{ml}$ directed spray 2 and $4 \mathrm{ml} \mathrm{L}$ against grasses and broad leaf weed control respectively as layby directed application. Cyanotis auxillaris, Commelina benghalensis and Digera arvensis were effectively controlled by Pyrithiobac sodium $1.8 \mathrm{ml} \mathrm{L}^{-1}$ or glyphosate $2 \mathrm{ml} \mathrm{L}^{-1}$. Commelina benghalesis was effectively controlled by both quizalofop-ethyl and fenoxoprop-methyl. Yellowing and stunted growth of cotton was observed with all the post emergence herbicides application compared to farmer's practice. Validation trials in farmers field's observed the yellowing was recovered by a fortnight later but farmers are forcibly using PGRs to fasten the early seedling growth, which is increasing the cost of cultivation by US $\$ 400 \mathrm{ha}^{-1}$. 


\section{Introduction}

Weed management involved $27 \%$ cost of cultivation of cotton due to recent increase in the manual labour costs in rural India, lead to uneconomical cotton cultivation (Reddy et al., 2013). Changing rainfall patterns after 2008, where both initial seedling droughts or incessant and continued late rains favour for high intensity and excess weed growth, which reduced seed cotton yields and doubled the weed management cost (Raju and Thakare, 2014). Early post emergence herbicide Pyrithiobac sodium under continuous rains in 2008 gave an adoption rate of $40 \%$ in the very $1^{\text {st }}$ year of its market launching. A silent revolution in weed management of rainfed cotton is under gone during 2005-2012 by adopting herbicides usage $35 \%$ (Gianessi, 2014), where farmers spent US \$16-36 $\mathrm{ha}^{-1}$ compared to US \$ $69 \mathrm{ha}^{-1}$ on manual weeding out of total cash inputs US \$965 ha ${ }^{-1}$ on cultivation of cotton (Reddy et al., 2013). Pyrithiobac sodium as early post emergence herbicide application alone failed to control grasses and some of those tolerant broad leaved weeds to Imzethapyr (ALS group) being applied since last 18 years in soybeancotton rotation (Raju and Thakare, 2014). This led to addition of quizalofop ethyl on trial and error basis or directed sprays of glyphosate with a hood in $B t$ hybrid cotton. Post emergence graminicides, fenoxoprop and propaquizafop herbicides were also commercially introduced in 1997 for wheat and soybean / potato respectively. Quizalofop ethyl, fenoxoprop and propaquizafop as tank mixture along with pyrithiobac sodium herbicides were also advocated by the pesticide dealers to soybean and cotton in the absence of official recommendations since 2007 (Kulkarni and Damodaran, 2010). Pendimethalin PPI application followed by once or twice roundup ultra application at 25 DAS in nut sedge infested fields provided good weed control in NR cotton with moderate intensity of weeds, where as pyrithiobac as post emergence application was sufficient to take care of low density weed populations. Present investigation in ICAR sponsored Technology Mission on Cotton was targeted to provide immediate low cost weed management solutions to replace costly manual weeding by adopting available officially non recommended herbicides for cotton from the market for heavily weed infested fields for NR cotton.

\section{Materials and Methods}

A field experiment was conducted in vertisols under rainfed narrow row (NR) cotton with C.var. Suraj at ICAR-CICR, Research farm, Nagpur. Twelve herbicide combinations in 6 replications were conducted in medium and shallow soils respectively during 2013, 2014 years in a year of excess wet monsoon and dry weather condition/seedling drought years respectively. Treatments executed were Control; Farmer's practice (three interculture followed by $(f b)$ two manual weedings); Quizalofop ethyl $5 \mathrm{ml} \mathrm{L} \mathrm{L}^{-1}$; Fenoxoprop methyl; Propaquizafop ethyl; Pyrithiobac sodium; Quizalofop ethyl + pyrithiobac sodium; Fenoxy prop methyl + pyrithiobac sodium $1.8 \mathrm{ml} \mathrm{L}^{-1}$; Propaquizafop ethyl + pyrithiobac sodium; Propaquizafop + pyrithiobac sodium $f b$ glyphosate $6 \mathrm{ml} \mathrm{L}^{-1} 60$ \& 90 DAS; Glyphosate $6 \mathrm{ml} \mathrm{L}^{-1} 35,60$ \& 90 DAS; Propaquizafop ethyl + pyrithiobac sodium, $f b$ glyphosate $6 \mathrm{ml} \mathrm{L}^{-1} 60 \& 90$ DAS during 2013; which differed in 2014 as Propaquizafop + pyrithiobac sodium $f b$ glyphosate $4 \mathrm{ml} \mathrm{ml} \mathrm{L}{ }^{-1} 60 \& 90$ DAS; Glyphosate $2 \mathrm{ml} \mathrm{L}^{-1} 35$ DAS $f b 6 \mathrm{ml} \mathrm{L}^{-1} 60$ \& 90 DAS; Propaquizafop ethyl + pyrithiobac sodium, $f b$ glyphosate $2 \mathrm{ml} \mathrm{L}^{-1} \& 6 \mathrm{ml} \mathrm{L}^{-1} 60$ $\& 90$ DAS in order to improve weed control efficiency of resistant grasses in row and reducing the damage to cotton the spray was directed with the help of hood. All the treatments were prior sprayed with $0.6 \mathrm{~kg}$ a.i. 
$\mathrm{ha}^{-1}$ each of glyphosate and pendimethalin after emergence of weeds and before planting of cotton to kill germinated weeds and also to get a residual effect. Post emergence herbicides and their combinations were also sprayed either over the top where as glyphosate was only directed spray with a protective hood. Treatments were designed to take care of high intensity of weeds under continuous, extended monsoon season rains and also verified in a year which began with seedling drought with limited rainfall. One insecticide spray for sucking pests and two for boll worms were sprayed on non $B t$ cotton improved American variety Suraj. Weed count was measured using a quadrant of 0.5 $\mathrm{m}^{2}$ at 4 spots, the data was converted to square root of $(\mathrm{X}+\mathrm{V} 0.5)$ before subjected to statistical analysis and biomass was expressed as $\mathrm{kg} \mathrm{m}^{-1}$ length. Weed control efficiencies were computed from total weeds $v s$ killed by herbicides and the values were converted to arcsin before statistical analysis. Economics was calculated using the prevailing market prices for cotton and herbicides such as glyphosate @ 4.6 quizalofop ethyl @ 15, fenoxy prop methyl @20, propaquizafop ethyl @ 23 pyrithiobac na $43 \mathrm{~L}^{-1}$, daily wages for male labourers@4.1 day ${ }^{-1}$, female @ 2.5 day $^{-1}$ and bullock power @US \$ 10 day $^{-1}$ respectively.

\section{Results and Discussion}

\section{Pendimethalin+glyphosate as PPF application on $\mathbf{1}^{\text {st }}$. flush of weeds}

Glyphosate+ pendimethalin 1:1 ratio each 1.0 $\mathrm{kg}$ a.i. ha-1 tank mixture as PPF resulted in very low incidence of tolerant weeds to either glyphosate or pendimethalin besides some second flush of weeds, which were germinated after 37 DAS, but killed very efficiently by glyphosate directed spray @ 1.0 $\mathrm{kg}$ a.i. ha ${ }^{-1}$.
Glyphosate PPF, pre and post emergence herbicides application

Graminaceous weeds Setaria intermedia, Cynotis auxilari, Cynodon dactylon and broad leaved weed Acalypha indica, Abutilon indicum, Convolvulus arvensis, Merremia imarginata and Tridax procumbence were not controlled by glyphosate + pendimethalin $1: 1$ ratio each $1.0 \mathrm{~kg}$ a.i. $\mathrm{ha}^{-1}$ tank mixture as $\mathrm{PPF}$ followed $f b$ post emergence herbicides propaquizafop ethyl, pyrithiobac sodium. These tolerant or escaped weeds need to remove by intercultural operations or hand weeding or directed application of glyphosate spray with the help of hood. Lowest weed biomass and highest weed control efficiency were produced by the treatments propaquizafop ethyl + pyrithiobac sodium, glyphosate $6 \mathrm{ml} \mathrm{L}^{-1}$. Highest weed control efficiency of broad leaves was achieved in propaquizafop + pyrithiobac sodium $f b$ glyphosate directed application at 60 DAS. These results were in agreement with those by Kulkarni and Damodaran, 2010 for pyrithiobac and roundup in cotton. The highest weed control efficiency of grasses was achieved with post emergence application of propaquizafop (Table 1 and 2).

\section{Post emergence herbicides alongwith intercultural operations in wet monsoon}

Three interculture operations in one direction were given on $9 / 8 / 13 ; 16 / 8 / 13$ and $2 / 9 / 13$ followed by two hand weedings in case of farmer's practice. Acalypha indica and Tridax procumbense and Merremia imarginata major broad leaved weeds which were not controlled by pyrithiobac sodium alone or in combination with quizalofop ethyl and glyphosate directed spray. However, other major weeds were Abutilon indicum, Cardiospermum sp. and Parthenium hysterophorus could be controlled by the same combination of herbicides. The best 
weed control efficiency of broad leaved weeds was achieved with pyrithiobac sodium, while all graminicides and their combinations were equally effective. Glyphosate alone directed spray in the cotton rows a practice some farmers followed on early grown up weeds under incessant rains was significantly lowest weed control efficiency.

\section{Conclusion for wet monsoon}

Glyphosate + pendimethalin 1:1 ratio each 1.0 $\mathrm{kg}$ a.i. ha ${ }^{-1}$ tank mixture as PPF application on the germinated weeds followed by glyphosate directed spray at 35, 60 and 90 DAS three times or glyphosate at 60,90 DAS or at 60 DAS replaced with propaquizafop + pyrithiobac sodium were most economical weed control options for heavily weed infested soils under wet monsoon conditions (Table 3).

\section{Weed management techniques for year of seedling drought}

A delay in one month of onset of monsoon rains and hot desiccating winds did not permit the weeds to germinate. Hence, residual herbicides pendimethalin @ $1.0 \mathrm{~kg}$ a.i. ha ${ }^{-1}$ along with PPF application of glyphosate @ $1.0 \mathrm{~kg}$ a.i. $\mathrm{ha}^{-1}$ each herbicide in $1: 1$ ratio tank mixture as PPF application on stale seed bed did not worked in a year of seedling drought.

Table.1 Weed control by post emergence herbicides application in wet monsoon

\begin{tabular}{|c|c|c|c|c|c|c|c|c|c|c|c|c|}
\hline \multirow[t]{3}{*}{ Treatments } & \multicolumn{4}{|c|}{$24 / 7 / 13$} & \multicolumn{4}{|c|}{$17 / 8 / 13$} & \multicolumn{4}{|c|}{$26 / 9 / 13$} \\
\hline & \multicolumn{2}{|c|}{ Grasses } & \multicolumn{2}{|c|}{ Broad leaved } & \multicolumn{2}{|c|}{ Grasses } & \multicolumn{2}{|c|}{ Broad leaved } & \multicolumn{2}{|c|}{ Grasses } & \multicolumn{2}{|c|}{ Broad leaved } \\
\hline & $\mathrm{O}$ & $\mathrm{C}$ & $\mathrm{O}$ & $\mathrm{C}$ & $\mathrm{O}$ & $\mathrm{C}$ & $\mathrm{O}$ & $\mathrm{C}$ & $\mathrm{O}$ & $\mathrm{C}$ & $\mathrm{O}$ & $\mathrm{C}$ \\
\hline Control & 9.0 & 2.66 & 5.3 & 2.29 & 1.3 & 2.7 & 1.0 & 1.77 & 8.0 & 2.9 & 3.0 & 1.78 \\
\hline $\begin{array}{l}\text { Three intercultures+2 hand } \\
\text { weedings }\end{array}$ & 0.0 & 0.71 & 0.0 & 0.71 & 0.3 & 0.7 & 3.0 & 0.71 & 7.3 & 2.8 & 2.3 & 1.64 \\
\hline Quizalofop ethyl $5 \mathrm{ml} \mathrm{L}^{-1}$ & 20.0 & 3.09 & 11.7 & 3.39 & 3.0 & 1.4 & 3.0 & 1.90 & 3.3 & 1.9 & 5.0 & 2.18 \\
\hline Fenoxy prop methyl $1.8 \mathrm{ml} \mathrm{L}^{-1}$ & 11.0 & 2.51 & 38.3 & 5.60 & 5.0 & 1.9 & 4.3 & 2.12 & 2.0 & 1.4 & 5.7 & 2.41 \\
\hline Propaquizafop ethyl $1.8 \mathrm{ml} \mathrm{L}^{-1}$ & 27.0 & 4.84 & 17.7 & 3.71 & 3.3 & 2.1 & 2.0 & 2.11 & 4.7 & 2.3 & 2.3 & 1.57 \\
\hline Pyrithiobac sodium $1.8 \mathrm{ml} \mathrm{L}^{-1}$ & 16.0 & 3.43 & 9.0 & 2.95 & 3.3 & 1.7 & 2.3 & 1.64 & 2.0 & 1.5 & 4.3 & 2.18 \\
\hline Quizalofop + Pyrithiobac $\mathrm{Na}$ & 9.0 & 2.99 & 4.7 & 2.16 & 2.3 & 1.8 & 4.0 & 1.61 & 0.0 & 0.7 & 5.0 & 2.30 \\
\hline Fenoxy prop + Pyrithiobac $\mathrm{Na}$ & 3.0 & 1.79 & 10.0 & 3.14 & 1.0 & 1.4 & 2.3 & 2.02 & 1.3 & 1.2 & 4.7 & 2.26 \\
\hline Propaquizafop + Pyrithiobac & 19.0 & 3.47 & 4.0 & 2.10 & 2.0 & 1.6 & 3.0 & 1.17 & 3.3 & 1.8 & 3.3 & 1.95 \\
\hline $\begin{array}{l}\text { Propaquiza fop + Pyrithiobac } 35 \\
\text { DAS } f b \text { Glyphosate } 6 \mathrm{ml} \mathrm{L}^{-1} 60 \\
\& 90 \text { DAS }\end{array}$ & 2.0 & 0.71 & 0.0 & 0.71 & 6.7 & 1.3 & 2.7 & 2.16 & 4.7 & 2.3 & 1.3 & 1.27 \\
\hline $\begin{array}{l}\text { Glyphosate } 6 \mathrm{ml} \mathrm{L}^{-1} 35,60 \& \\
90 \text { DAS }\end{array}$ & 15.0 & 3.48 & 0.7 & 1.05 & 8.7 & 2.8 & 0.7 & 1.34 & 6.7 & 2.5 & 3.7 & 2.04 \\
\hline $\begin{array}{l}\text { Glyphosate } 6 \mathrm{ml} \mathrm{L}^{-1} 35,90 \& \\
\text { Propaquizafop }+ \text { Pyrithiobac } \\
\text { sodium } 60 \text { DAS }\end{array}$ & 15.0 & 3.34 & 2.3 & 1.68 & 13.3 & 2.9 & 12.7 & 1.17 & 6.7 & 2.6 & 2.7 & 1.76 \\
\hline $\mathrm{SED} \pm 5 \%$ & 12.2 & 1.5 & 8.6 & & 4.2 & & 3.4 & & 1.4 & 2.9 & 1.2 & 0.47 \\
\hline $\mathrm{CD} \pm 5 \%$ & & & & 2.43 & & 0.99 & & 0.85 & & 1.1 & & NS \\
\hline
\end{tabular}

$\mathrm{O}=$ original $\mathrm{C}=\arcsin$ converted values 
Table.2 Weed control efficiency by post emergence application of herbicides in wet monsoon

\begin{tabular}{|c|c|c|c|c|c|c|c|c|c|c|c|c|}
\hline \multirow[t]{3}{*}{ Treatments } & \multicolumn{4}{|c|}{$24 / 7 / 13$} & \multicolumn{4}{|c|}{$17 / 8 / 13$} & \multicolumn{4}{|c|}{$26 / 9 / 13$} \\
\hline & \multicolumn{2}{|l|}{ GR } & \multicolumn{2}{|l|}{ BL } & \multicolumn{2}{|l|}{ GR } & \multicolumn{2}{|l|}{ BL } & \multicolumn{2}{|l|}{ GR } & \multicolumn{2}{|l|}{ BL } \\
\hline & $\mathbf{O}$ & ASIN & $\mathbf{O}$ & ASIN & $\mathbf{O}$ & ASIN & $\mathbf{O}$ & ASIN & $\mathbf{O}$ & ASIN & $\mathbf{O}$ & ASIN \\
\hline Control & 0.04 & 0.16 & 0.30 & 0.33 & 0.42 & 0.97 & -0.67 & -1.05 & -0.67 & -0.67 & 0.50 & 0.50 \\
\hline Three intercultures+2 hand weedings & 1.00 & 1.57 & 0.89 & 1.09 & 1.00 & 1.43 & 1.00 & 1.57 & -0.75 & -0.77 & 0.61 & 0.60 \\
\hline Quizalofop ethyl $5 \mathrm{ml} \mathrm{L}^{-1}$ & 0.33 & 0.52 & -0.33 & -0.52 & 0.86 & 1.10 & -0.33 & -0.52 & 0.17 & 0.17 & 0.17 & 0.20 \\
\hline Fenoxy prop methyl $1.8 \mathrm{ml} \mathrm{L}^{-1}$ & 0.30 & 0.36 & -0.67 & -1.05 & 0.75 & 0.64 & -1.00 & -1.57 & 0.50 & 0.50 & 0.06 & 0.03 \\
\hline Propaquizafop ethyl $1.8 \mathrm{ml} \mathrm{L}^{-1}$ & -0.48 & -0.85 & -0.19 & -0.34 & 0.64 & 0.73 & -1.00 & -1.57 & -0.17 & -0.17 & 0.61 & 0.60 \\
\hline Pyrithiobac sodium $1.8 \mathrm{ml} \mathrm{L}^{-1}$ & 0.07 & -0.07 & -0.11 & -0.30 & 0.72 & 1.06 & -0.33 & -0.52 & 0.50 & 0.50 & 0.28 & 0.27 \\
\hline Quizalofop + Pyrithiobac Na & -0.04 & -0.21 & 0.37 & 0.41 & 0.75 & 0.88 & 0.00 & 0.00 & 1.00 & 1.00 & 0.17 & 0.17 \\
\hline Fenoxy prop + Pyrithiobac Na & 0.63 & 0.76 & -0.22 & -0.28 & 0.83 & 1.24 & -0.67 & -1.05 & 0.67 & 0.67 & 0.22 & 0.23 \\
\hline Propaquizafop + Pyrithiobac & -0.15 & -0.33 & 0.44 & 0.47 & 0.83 & 1.06 & 0.00 & 0.00 & 0.17 & 0.13 & 0.44 & 0.43 \\
\hline $\begin{array}{l}\text { Propaquiza fop + Pyrithiobac } 35 \text { DAS } \\
f b \text { Glyphosate } 6 \mathrm{ml} \mathrm{L}^{-1} 60 \text { \& } 90 \text { DAS }\end{array}$ & 1.00 & 1.57 & 0.89 & 1.09 & 0.89 & 0.77 & -0.67 & -1.05 & -0.17 & \begin{tabular}{|l|l|}
-0.17 \\
\end{tabular} & 0.78 & 0.77 \\
\hline Glyphosate $6 \mathrm{ml} \mathrm{L}^{-1} 35,60 \& 90$ DAS & -0.26 & -0.45 & 0.81 & 0.96 & 0.36 & 0.18 & 0.67 & 1.05 & -0.17 & -0.17 & 0.39 & 0.37 \\
\hline $\begin{array}{l}\text { Glyphosate } 6 \mathrm{ml} \mathrm{L}^{-1} 35,90 \& \text { Propaquizafop + } \\
\text { Pyrithiobac sodium } 60 \text { DAS }\end{array}$ & -0.19 & -0.18 & 0.63 & 0.68 & 0.28 & 0.66 & 0.67 & 1.05 & -0.58 & -0.60 & 0.56 & 0.57 \\
\hline SED $\pm 5 \%$ & 0.06 & 0.88 & 0.08 & & 0.23 & & -0.06 & & 0.01 & & 0.13 & 0.33 \\
\hline $\mathrm{CD} \pm \overline{5} \%$ & & & & 1.18 & & 0.58 & & 1.99 & & 0.91 & & NS \\
\hline
\end{tabular}

Table.3 Economics of post emergence herbicides in wet monsoon

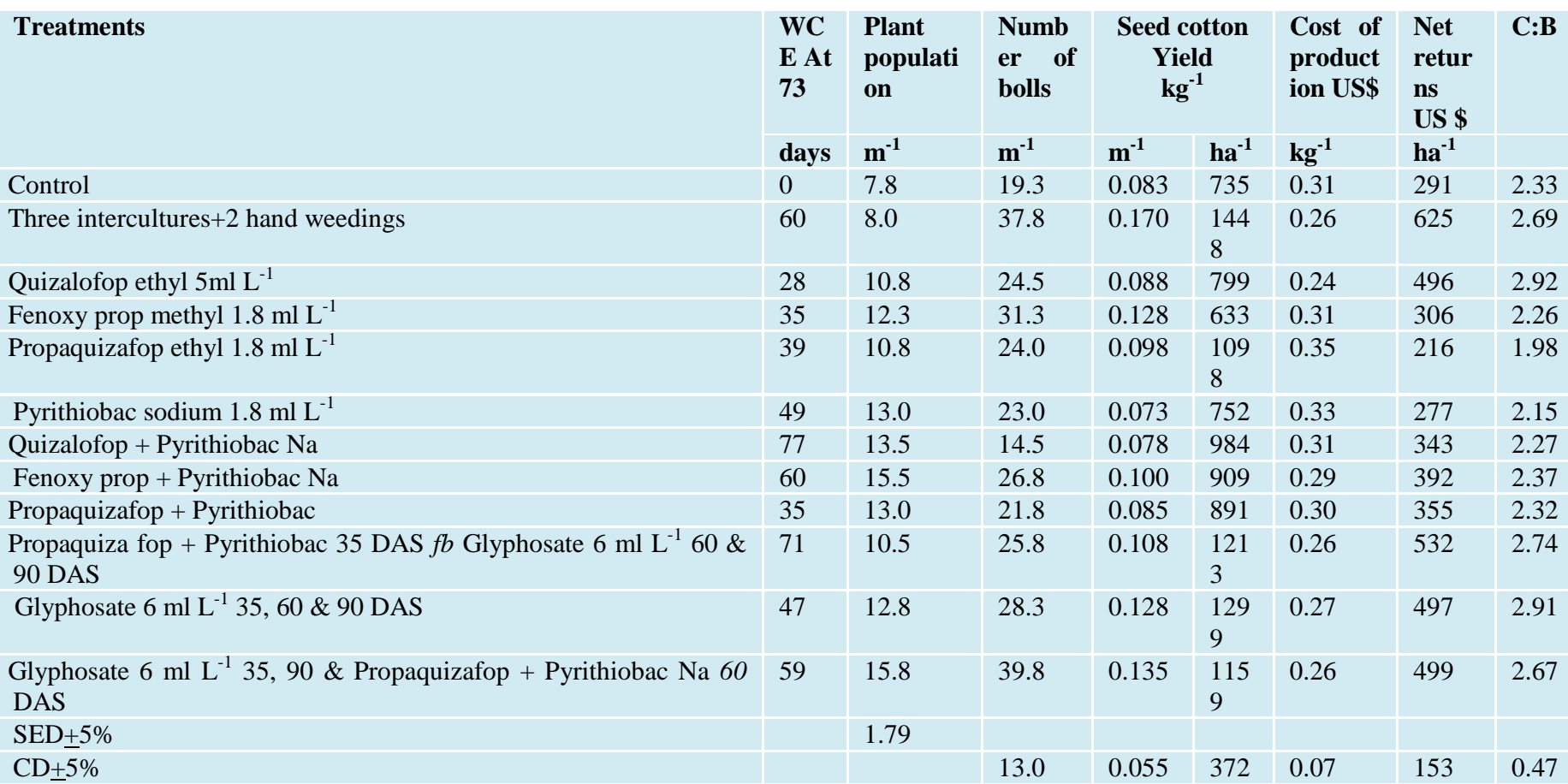


Table.4 Major weeds controlled by post emergence herbicides application in drought year

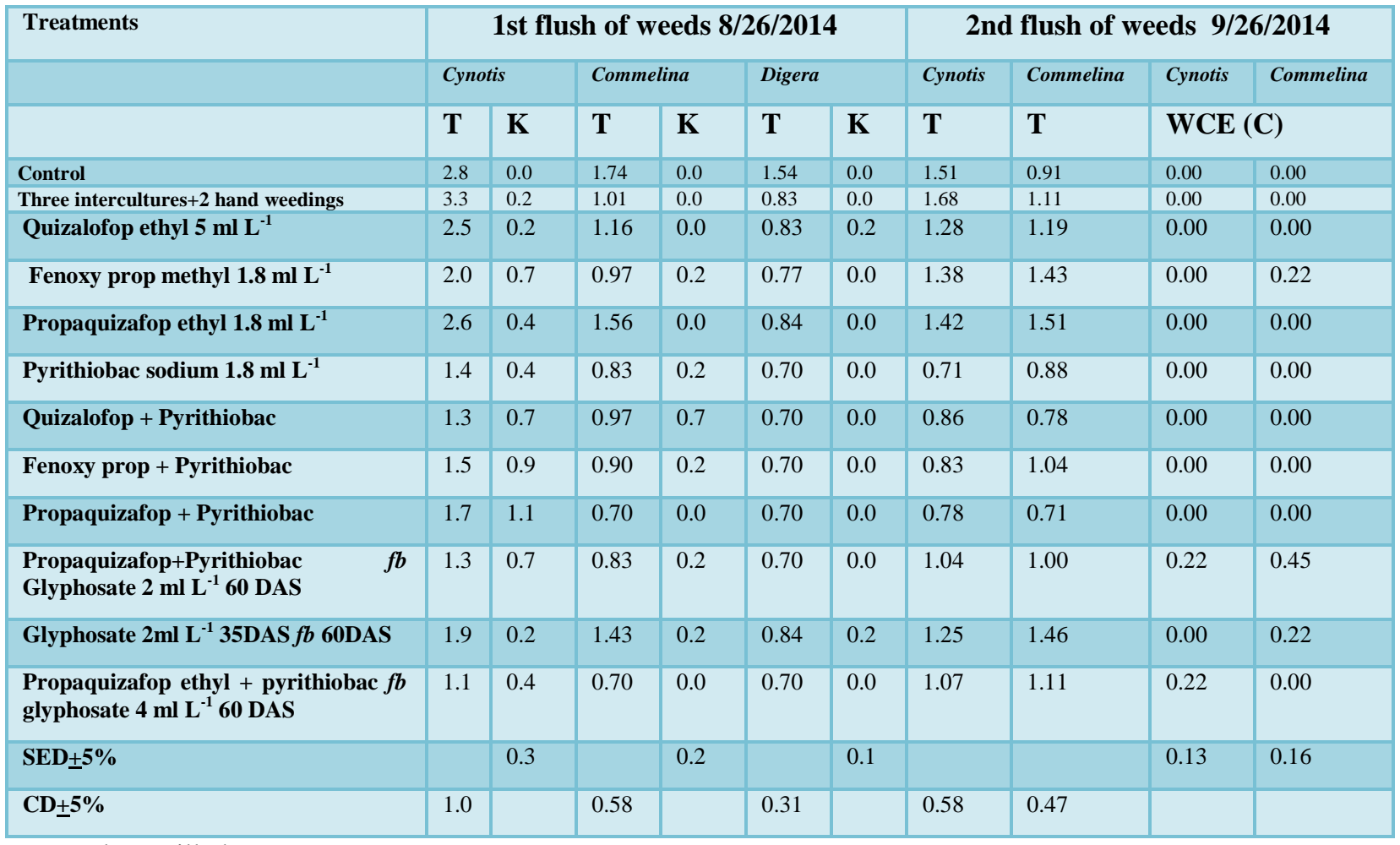

$\mathrm{T}=$ Total $\mathrm{K}=$ Killed

Table.5 Weed BM, WU, WUE and WCE by post emergence herbicides application in drought year

\begin{tabular}{|c|c|c|c|c|c|c|c|c|}
\hline \multirow[t]{2}{*}{ Treatments } & \multicolumn{2}{|c|}{ Soil moisture use } & \multicolumn{3}{|c|}{ Weed BM Kg m ${ }^{-2}$} & \multicolumn{3}{|c|}{ WCE 8.10.14 } \\
\hline & Water use & WUE & 26.8.14 & 26.9.14 & 9.10 .14 & BL & GR & Mean \\
\hline Control & 34.7 & 2.7 & 0.051 & 0.029 & 0.055 & 0 & 0 & \\
\hline Three intercultures +2 hand weedings & 34.0 & 19.1 & 0.018 & 0.027 & 0.000 & & & \\
\hline Quizalofop ethyl $5 \mathrm{ml} \mathrm{L}^{-1}$ & 28.3 & 23.0 & 0.025 & 0.022 & 0.046 & 15 & 4 & 10 \\
\hline Fenoxy prop methyl $1.8 \mathrm{ml} \mathrm{L}^{-1}$ & 28.3 & 27.0 & 0.037 & 0.022 & 0.039 & 18 & 16 & 17 \\
\hline Propaquizafop ethyl $1.8 \mathrm{ml} \mathrm{L}^{-1}$ & 32.7 & 13.7 & 0.043 & 0.020 & 0.040 & 0 & 37 & 19 \\
\hline Pyrithiobac sodium $1.8 \mathrm{ml} \mathrm{L}^{-1}$ & 30.0 & 14.4 & 0.048 & 0.047 & 0.043 & 33 & 3 & 18 \\
\hline Quizalofop + Pyrithiobac & 25.3 & 27.1 & 0.019 & 0.037 & 0.039 & 65 & 85 & 75 \\
\hline Fenoxy prop + Pyrithiobac & 22.7 & 13.6 & 0.029 & 0.029 & 0.045 & 58 & 50 & 54 \\
\hline Propaquizafop + Pyrithiobac & 25.0 & 22.1 & 0.014 & 0.025 & 0.044 & 63 & 48 & 55 \\
\hline $\begin{array}{l}\text { Propaquizafop+Pyrithiobac } f b \text { Glyphosate } 2 \\
\text { ml L }^{-1} 60 \text { DAS }\end{array}$ & 23.7 & 27.1 & 0.025 & 0.022 & 0.033 & 58 & 73 & 66 \\
\hline Glyphosate $2 \mathrm{ml} \mathrm{L}^{-1}$ 35DAS $f b$ 60DAS & 20.3 & 10.7 & 0.035 & 0.028 & 0.058 & 50 & 58 & 54 \\
\hline $\begin{array}{l}\text { Propaquizafop ethyl }+ \text { pyrithiobac } f b \\
\text { glyphosate } 4 \mathrm{ml} \mathrm{L}^{-1} 60 \text { DAS }\end{array}$ & 19.0 & 18.5 & 0.033 & 0.025 & 0.046 & 46 & 100 & 73 \\
\hline SED $\pm \mathbf{5} \%$ & & 8.5 & & & & & & \\
\hline$C D \pm 5 \%$ & 5.7 & & 0.025 & 0.023 & 0.022 & & & \\
\hline
\end{tabular}


The major weeds Cynotis auxillaris, Commelina benghalensis and Digera arvensis were analysed based on their distribution and weed kill as per standard protocol on $26^{\text {th }}$ of August and September, $9^{\text {th }}$. October months and found pyrithiobac sodium and glyphosate directed spray of $4 \mathrm{ml} \mathrm{L}^{-1}$ could control all the weeds. However, quizalofop and fenoxoprop could not control Cynotis auxillaris. Similar trends were observed for weed control efficiency on $2^{\text {nd }}$.

Flush of weeds with yellowing when the weeds grown beyond a month, where glyphosate directed spray was more effective than other post emergence herbicides. Propaquizafop ethyl + pyrithiobac sodium $f b$ glyphosate@ $2 \mathrm{ml} \mathrm{L}^{-1} f b 4 \mathrm{ml} \mathrm{L}^{-1} 35,60$ DAS, quizalofop ethyl + pyrithiobac sodium and glyphosate $2 \mathrm{ml} \mathrm{L}^{-1} 35$ DAS $f b 60$ DAS glyphosate $6 \mathrm{ml} \mathrm{L}^{-1}$ produced acceptable range of broad leaved weed control efficiency 85,75 and $72 \%$ respectively (Table 4 and 5). Propaquiza fop + pyrithiobac $f b$ glyphosate 2 $\mathrm{ml} \mathrm{\textrm {L } ^ { - 1 }} 60$ DAS only produced acceptable grassy weed control of $90 \%$ followed by alone or its combinations with glyphosate around 50\% weed control efficiency (Table 4 and 5). Propaquizafop + pyrithiobac sodium $f b$ glyphosate $2 \mathrm{ml} \mathrm{L}^{-1} 60$ DAS produced lowest weed biomass best water use efficiency and weed control efficiency (Table 5).

It is concluded that in a year of weak monsoon farmers can go direct sowing of cotton when $60 \mathrm{~mm}$ rain is received without any pre emergence residual herbicide application of pendimethalin @ $1.0 \mathrm{~kg}$ a.i. ha ${ }^{-1}$ with seedling drought. The year may begin with very good monsoon can have only glyphosate @ $1.0 \mathrm{~kg}$ a.i. ha ${ }^{-1}$ as PPF application on emerged weeds followed on or before planting of cotton. The year might began with very good monsoon with seedling drought when interculture operations can take care of weeds followed by limited hand weedings with available family/hired labour. However, revival of monsoon or late continuous heavy rains farmers can use propaquizafop the best graminicide alone or along with pyrithiobac sodium for broad leaf weeds. In case late active monsoon rains after squaring stage when rains farmers may use glyphosate $2 \mathrm{ml}$ directed spray on grasses and $4 \mathrm{ml} \mathrm{L}$ on broad leaves weed control as late application on younger weeds which are difficult to be removed by hand weeding operations.

\section{Acknowledgment}

Authors have duly acknowledge the financial assistance received under Development of Agro-technologies for $G$. hirsutum $G$. arboreum in HDPS cotton TMC MM 1.4; 2012-2016; Department of Agriculture and Cooperation, New Delhi through ICAR, New and Central Institute for Cotton Research, Nagpur

\section{References}

Gianessi, L., 2013. Income and yield benefits driving large increase in herbicide use in India. International pesticide benefits case study No. 93, November 2013. Crop Protection Research Institute. https://croplife.org/wpcontent/uploads/2 014/05/ income-and-yield-benefitsdriving-large-increase-in-herbicideuse-in-India.pdf.

Kulkarni, V. and Damodaran, H., 2010. Dhanuka in talks with 2 global pesticide majors for possible JVs. The Hindu Business line. Dec. $1^{\mathrm{st}}$, http:// www.thehindubusinessline.com/industr y-and-economy/agri-biz/dhanuka-intalks-with-2-global-pesticide-majorsfor-possible-jvs/article2677964.ece

Habimana S.,. Kalyana murthy, K., N., Shankaralingappa, B., C., Devendra, R., Sanjay M., T. and Ramachandra, C. 
2013. Effect of pre and post-emergence herbicides on weed dynamics, growth and yield of soybean (Glycine Max L.). Advances in Applied Science Research,2013,4(4):72-7.

Raju, A.R. 2014. Improving hybrid cotton profitability and micronaire with strip cropping of soybean + pigeon pea with conservation furrows, $\mathrm{Mn}$ and $\mathrm{B}$ application. African J. of Agric Res. Vol. 9(2):183-195.DOI $10.5897 /$
AJAR2013.7331

Raju, A.R. Soniya K. Thakare, G. Majumdar and P.R. Bharambe, 2013. Risk aversion in shallow soils with innovative intercropping systems, $J$. of Cotton Res Dev., 27(1):37-44.

Reddy, A.R., Singandhupe R., B. and Rokde, S., N. 2013. Impact evaluation of $\mathrm{Bt}$ cotton in Maharashtra. Draft report submitted to Dept. Of Agriculture, Govt. of Maharashtra state, India.

\section{How to cite this article:}

Ambati Ravinder Raju, Shilpa Rananware and Rachana Deshmukh. 2018. Pre and Post emergence Cotton Herbicides in Varying Rainfall Patterns. Int.J.Curr.Microbiol.App.Sci. 7(08): 3637-3644. doi: https://doi.org/10.20546/ijcmas.2018.708.368 